\title{
Reduction in rectal doses of pelvic radiotherapy with excessive rectal gas extraction: a single-institution case report
}

\author{
Xue Jiang ${ }^{1}$, Ying Chen ${ }^{1}$, Junqing Liu ${ }^{1}$, Lihua Ning ${ }^{1}$, Xiaoli Sun ${ }^{1}$, Linyan Zheng ${ }^{1}$, Xinke Li ${ }^{1}$, Fang Wang ${ }^{1}$, \\ Xinli Zhu ${ }^{1}$, Yufeng Lou ${ }^{2}$, Senxiang Yan $^{1}$ \\ ${ }^{1}$ Department of Radiation Oncology, the First Affiliated Hospital, College of Medicine, Zhejiang University, Hangzhou, China; ${ }^{2}$ Department of \\ Hepatopancreatobiliary Surgery, the First Affiliated Hospital, College of Medicine, Zhejiang University, Hangzhou, China \\ Correspondence to: Senxiang Yan. Department of Radiation Oncology, the First Affiliated Hospital, College of Medicine, Zhejiang University, \\ Hangzhou 310003, China. Email: yansenxiang@zju.edu.cn.
}

\begin{abstract}
During radiotherapy for pelvic tumors, there might be insufficient radiation doses delivered that result from concerns of immediately adjacent critical organs, most notebly the rectum. Several measures have been proposed to reduce the rectal doses, such as use of a radioprotective spacer to shift the rectum away from the lesion or an endorectal balloon to reduce the internal target volume (ITV). The removal of rectal gas is supposed to achieve the similar effect by reducing the rectal volume and ITV. This is because the distance of the rectum to lesions can be increased with decreasing rectal volumes, which also makes it easy for physicists to avoid the rectum during field design. Besides, a reduced ITV can be obtained due to stability in rectal volume, as with use of an endorectal balloon. The procedure is most suitable for patients receiving stereotactic body radiotherapy (SBRT) and high dose rate brachytherapy (HDR-BR) because of limited treatment sessions. In this study, we showed that the rectal dose decreased significantly with rectal gas extraction, as manifested by dose parameters of $\mathrm{D} 2 \mathrm{~cm}^{3}$ and $\mathrm{D} 0.1 \mathrm{~cm}^{3}$ (the doses delivered to the most exposed $2 \mathrm{~cm}, 3$ and $0.1 \mathrm{~cm}^{3}$ of the rectum, respectively). This technique is especially benefitable for candidates with excessive rectal gas fillings.
\end{abstract}

Keywords: Rectal dose reduction; rectal gas; stereotactic body radiotherapy (SBRT); high dose rate brachytherapy (HDR-BR); case report

Submitted May 01, 2020. Accepted for publication Nov 23, 2020.

doi: 10.21037/apm-20-1063

View this article at: http://dx.doi.org/10.21037/apm-20-1063

\section{Introduction}

Radiation therapy (RT) is one of the most effective treatment modalities for a wide range of malignancies, about $70 \%$ of cancer patients are treated with RT during the disease course. Stereotactic body radiotherapy (SBRT) yields excellent control rates for many types of solid malignancies, especially in oligometastatic and metastatic tumors $(1,2)$. High dose rate brachytherapy (HDR-BR); with the advantage of precise positioning of the source, infinitely variable dwell times/ positions, and shorter treatment time, is widely used to treat gynecological malignancies (3-5). HDR-BR serves to boost the gross tumor and has been confirmed to improve tumor control and survival rates $(6,7)$. However, extreme caution should be taken not to over-irradiate the adjacent organs at risk (OARs) because of high fractionation size in SBRT and HDR-BT.

The rectum is one of the important OARs, which should be strictly protected during pelvic radiotherapy. Radiationinduced rectal toxicities such as inflammation, bleeding, and even rectovaginal fistula formation, will impair the patient's quality of life and impact the course of treatment. Furthermore, the variable gas filling in the rectum adds difficulty to the radiotherapy treatment planning. Related studies (8) have shown that in as many as $35 \%$ of cases, gas accumulation occurs for the entire course of RT. Several methods have been proposed to reduce rectal dose rates clinically, such as use of a radioprotective spacer to shift the rectum away from the lesion or an endorectal balloon 
Table 1 Patient characteristics

\begin{tabular}{llllll}
\hline Case & Disease & Stage & Age & Gender & Radiotherapy method \\
\hline 1 & Prostate cancer & Metastatic (IV) & 61 & Male & SBRT \\
2 & Endometrial cancer & Early (IA) & 60 & Female & HDR-BR \\
3 & Cervical cancer & Locally advanced (IIB) & 53 & Female & HDR-BR \\
\hline
\end{tabular}

HDR-BR, high dose rate brachytherapy; SBRT, stereotactic body radiotherapy.

to reduce the internal target volume (ITV) (9-11). Because currently in China both of the above techniques are widely unavailable, we alternatively hypothesized that removal of the rectal gas can achieve the similar effect by reducing the rectal volume and ITV. This is because the distance of the rectum to lesions can be increased with decreasing rectal volumes, which also makes it easy for the physicists to avoid the rectum during field arrangement. Besides, a reduced ITV can be obtained due to constancy in the rectal volume, as with use of an endorectal balloon.

We present the following article in accordance with the Case Series reporting checklist (available at http://dx.doi. org/10.21037/apm-20-1063).

\section{Methods}

Three cases of patients with pelvic malignancies receiving SBRT or HDR-BT were retrospectively analyzed in single center. Our institution is a comprehensive hospital integrating teaching, scientific research and clinical practice. The case report is presented following the CARE Guidelines (12). All patients underwent simulation computed tomography (sim-CT) scans with proper bladder and bowel preparations prior to either SBRT or HDR-BT. After the first acquisition of CT images, excessive gas in the rectum was confirmed and subsequently extracted by inserting a catheter into the rectum. Then, a second simCT was taken, and together with the first one transported to the radiotherapy treatment planning system (TPS). Both SBRT and HDR-BT plans were made based on the two sim-CTs, and compared using dose-volume histograms $(\mathrm{DVH})$. The patients had no discomfort after a catheter in section. We further present the clinical factors, treatments, ages, and stages of the patients in Table 1.

All procedures performed in studies involving human participants were in accordance with the ethical standards of the institutional and/or national research committee(s) and with the Helsinki Declaration (as revised in 2013). Written informed consent was obtained from the patient.

\section{Case 1}

A 61-year-old man with prostate cancer had received pelvic radiation for five years after surgery. At the time of admission, the patient had multiple bone metastases with continuous endocrine therapy and was diagnosed as stage IV. Before admission, the patient was found to have pain in their left lower limb and left hip. A pelvic magnetic resonance imaging (MRI) scan revealed a softtissue mass near the left piriformis muscle. A positron emission computed tomography (PET-CT) scan suggested there to be a high metabolism rate; therefore, a new local metastasis was considered. The use of a local lesion SBRT for palliative and analgesic treatment was planned, and the prescribed dose was 2,400 cGY/3F, $800 \mathrm{cGY} / \mathrm{f}$, $5 \mathrm{~F} / \mathrm{W}$. The sim-CT (Figure 1) scan found the rectum to be significantly inflated after the patient's stool was drained. The closest point of the rectal mucosa to the tumor target was $0.1 \mathrm{~cm}$, as shown by the yellow arrow in Figure $1 \mathrm{~A}$. After a single prescription dose of $800 \mathrm{cGY}$ was given to the target planning target volume (PTV), the dose $\mathrm{D} 2 \mathrm{~cm}^{3}$ of rectal was as high as $662 \mathrm{cGY}$. Considering that the patient had a history of pelvic radiotherapy, the rectal reaction of the patient was severe after direct treatment was administered; therefore, the rectal dose needed to be urgently reduced. During SBRT treatment, catheters were inserted into the rectum after defecation, and the excess gas in the rectum was extracted using a $50 \mathrm{~mL}$ syringe after each treatment. The radiotherapy plan was reformulated to treat the patient according to the real-time rectal volume. Table 2 shows the data diagram of the rectal irradiation doses after rectal gas was extracted during the first three SBRT treatments. The pictures of the first three SBRT treatments are shown in Figure $1 B, C, D$. Next, we calculated the distance between the rectal mucosa and the target volume after the rectal gas was extracted. We then compared the results with the sim-CT (Figure 1A), which was widened to $2.04,2.0$, and $2.14 \mathrm{~cm}$ (as shown by the yellow arrow Figure $1 B, C, D$ ). The patient's rectal D2 $\mathrm{cm}^{3}$ dose was reduced to $385 \mathrm{cGY}$ (Figure $1 B$ ), 

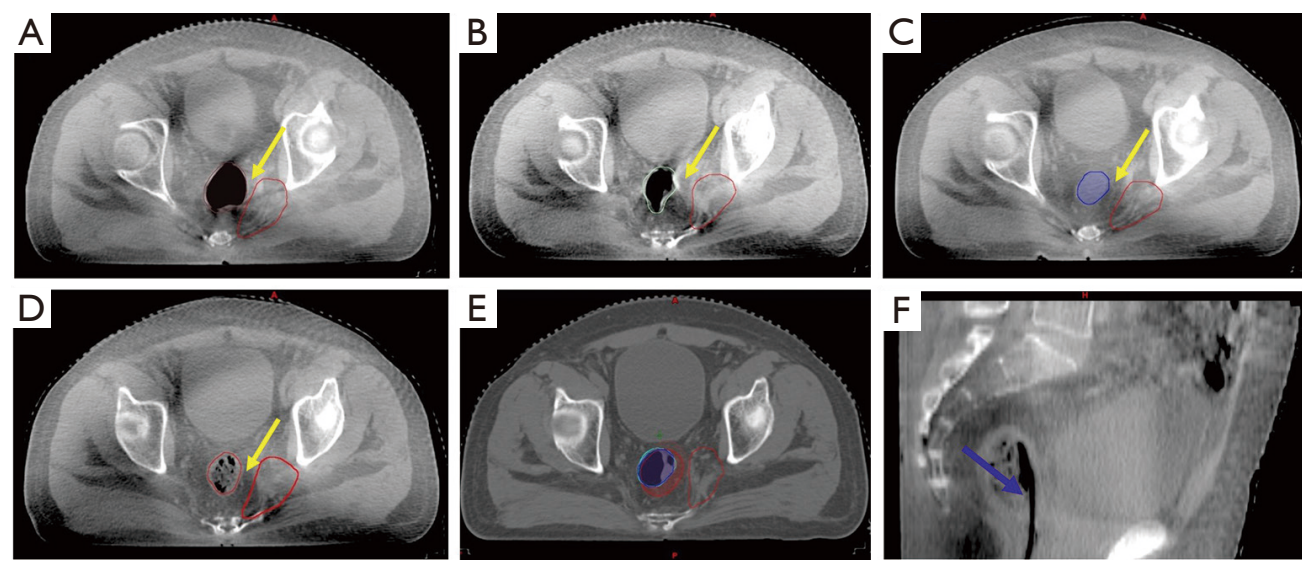

Figure 1 Changes of rectal volume and distance between rectum and tumor target area before and after extraction of gas by catheter in different SBRT groups. (A) shows the relationship between the rectum and the target area during the CT positioning, which is the initial image with no catheter insertion. The yellow arrow indicates a gap of $0.1 \mathrm{~cm}$. (B,C,D) The SBRT images obtained three times after rectal gas was extracted. The yellow arrow represents the widened distance. (E) The relationship between the four images of the rectum and the target area after fusing the images. (F) The image of the rectum's sagittal plane after the insertion of the catheter. The blue arrow shows the catheter. SBRT, stereotactic body radiotherapy.

Table 2 The rectal dose values and decline rates were compared between the initial CT scan and then after the gas extraction from the rectum

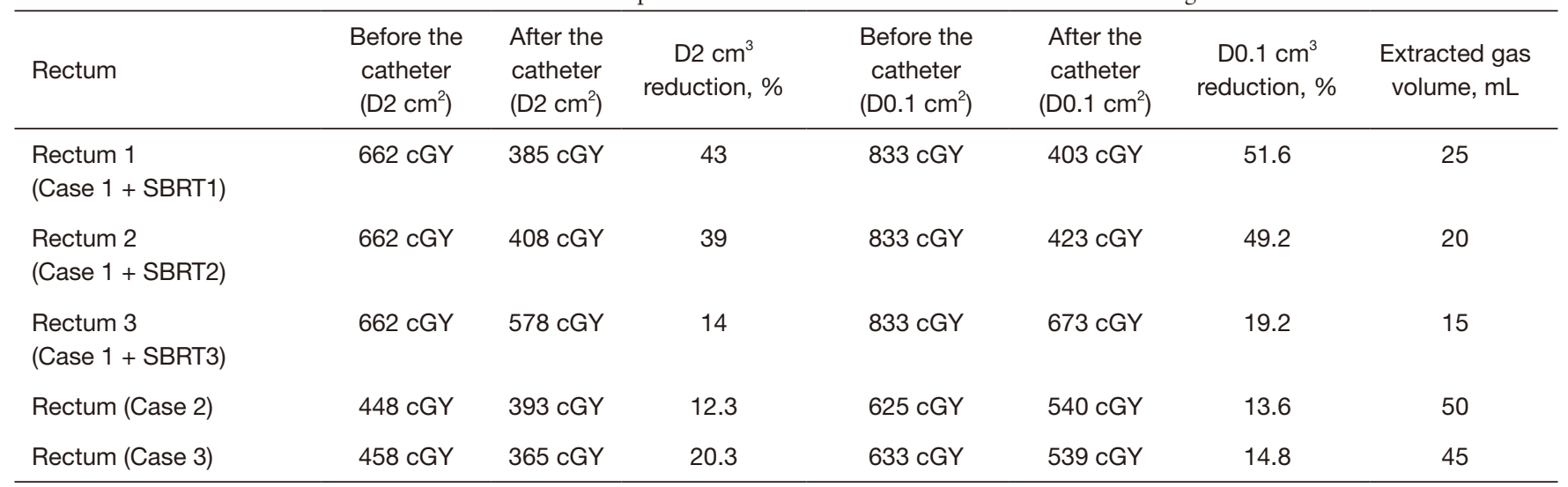

The last column is the amount of gas extracted. SBRT, stereotactic body radiotherapy.

408 cGY (Figure 1C), and 578 cGY (Figure 1D) (Table 2). Rectal D0.1 $\mathrm{cm}^{3}$ dose was reduced to $403 \mathrm{cGY}$ (Figure $1 B$ ), $423 \mathrm{cGY}$ (Figure 1C), and $673 \mathrm{cGY}$ (Figure 1D). The DVH of the rectal irradiation dose is shown in Figure 2. Compared with the sim-CT image without the catheter (Figure $1 A$ ), the patient's rectal D2 $\mathrm{cm}^{3}$ dose reduction was $43 \%, 39 \%$, and $14 \%$, respectively. The rectal D $0.1 \mathrm{~cm}^{3}$ dose reduction was $51.6 \%, 49.2 \%$, and $19.2 \%$, respectively (Table 2).

\section{Case 2}

The patient, female, 60 years old, was diagnosed with poorly differentiated endometrioid adenocarcinoma. The postoperative pathological stage was IA. The patient had several high-risk factors after surgery, prompting an HDRBT of the vaginal stump. For postoperative high-doserate vaginal brachytherapy, we preferred regimens include $5.5 \mathrm{GY}^{*} 4$ fractions prescribed to $5 \mathrm{~mm}$ below the vaginal 


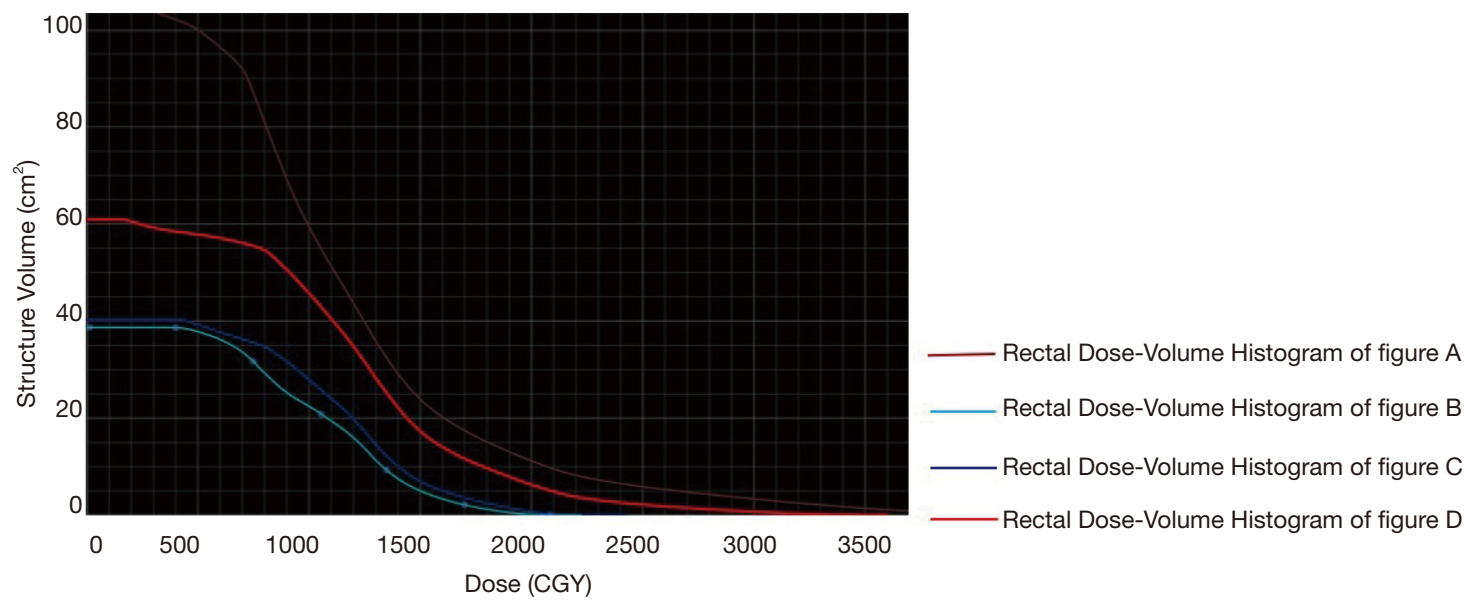

Figure 2 DVH of rectal irradiation dose in four SBRT radiotherapy plans. DVH, dose-volume histogram; SBRT, stereotactic body radiotherapy.
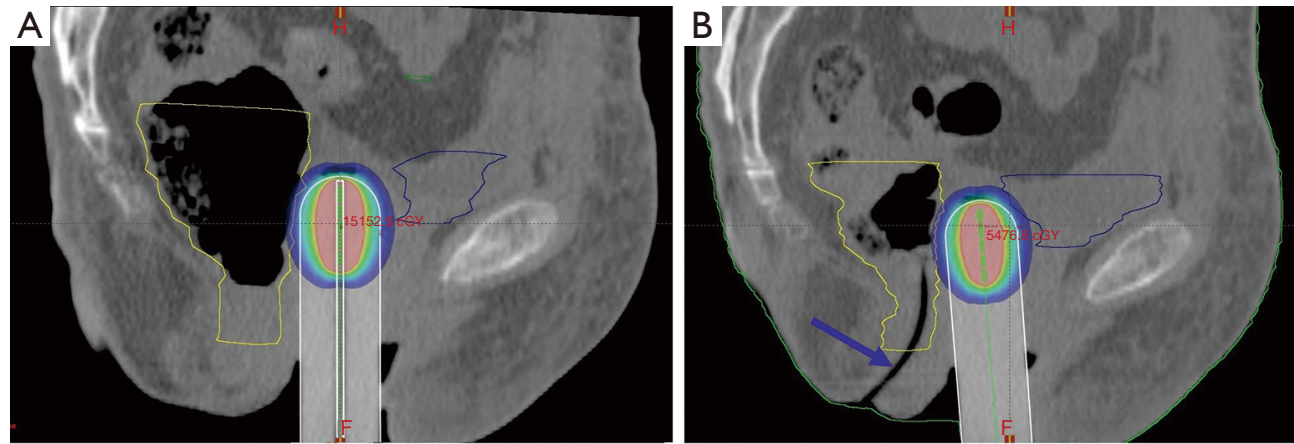

Figure 3 Rectal volume before and after insertion of a catheter. (A) The relationship between the significantly expanded rectum and the target volume, before the insertion of the catheter. (B) The relationship between the rectum and the target volume after the extraction of $50 \mathrm{~mL}$ gas. The blue arrow represents the catheter.

surface in our department (NCCN 2020). The rectum dosage was evaluated at $\mathrm{D} 2 \mathrm{~cm}^{3}$ and $\mathrm{D} 0.1 \mathrm{~cm}^{3}$. The patient had excessive gas before the insertion of the catheter (Figure $3 A$ ). $50 \mathrm{~mL}$ of gas was extracted by inserting the catheter, as shown by the blue arrow in Figure $3 B$. The prescription dose in the target volume remained unchanged, and the rectal doses required in Figure $3 A, B$ were calculated. D2 $\mathrm{cm}^{3}$ was $448 \mathrm{cGY}$ and $393 \mathrm{cGY}$, and D0.1 $\mathrm{cm}^{3}$ was $625 \mathrm{cGY}$ and $540 \mathrm{cGY}$, respectively. The dosimetry of D2 $\mathrm{cm}^{3}$ and D $0.1 \mathrm{~cm}^{3}$ decreased by $12.3 \%$ and $13.6 \%$, respectively, after the catheter was inserted (Table 2).

\section{Case 3}

The patient, a 53-year-old female, was admitted to the radiotherapy department after being diagnosed with cervix middle differentiated squamous cell carcinoma (stage IIB). The patient was treated with external irradiation plus threedimensional brachytherapy. HRCTV D90 600 cGY was given as a single prescription dose for BR treatment. The individual was treated with CT-guided three-dimensional brachytherapy, and the rectum dose was evaluated with D2 $\mathrm{cm}^{3}$ and D0.1 $\mathrm{cm}^{3}$. The CT scan of the patient revealed a slight increase in rectal gas, as shown in Figure 4A. A catheter was inserted, and $45 \mathrm{~mL}$ of gas was extracted with a syringe. The CT image was then rescanned (Figure $4 B$ ). The prescribed dose of HRCTV D90 600 cGY was given, the dose of rectal irradiation was D2 $\mathrm{cm}^{3}$ was $458 \mathrm{cGY}$ and $365 \mathrm{cGY}$, respectively. Simultaneously, D0.1 $\mathrm{cm}^{3}$ was $633 \mathrm{cGY}$ and $539 \mathrm{cGY}$, respectively. The dosimetry of D2 $\mathrm{cm}^{3}$ and D $0.1 \mathrm{~cm}^{3}$ decreased by $20.3 \%$ and $14.8 \%$, respectively, after the catheter was inserted (Table 2). 

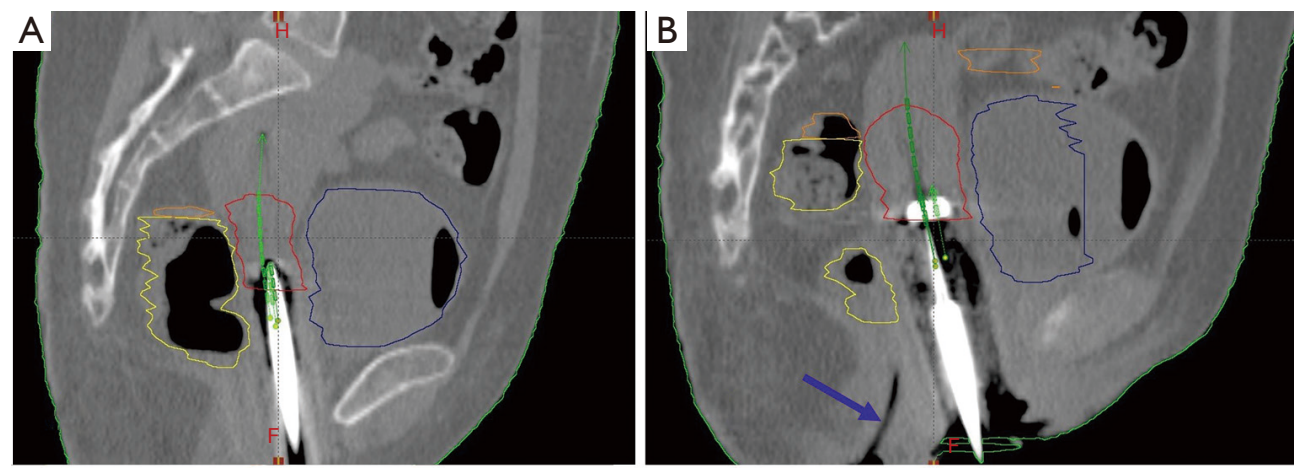

Figure 4 Rectal volume before and after insertion of a catheter. (A) The rectal volume before the insertion of the catheter. The rectum is filled with gas. (B) An image of $45 \mathrm{~mL}$ of gas extracted after the insertion of the catheter. The blue arrow represents the catheter.

\section{Discussion}

Although patients were recommended not to eat less gasproducing food during treatment, we still find that many patients can accumulate large quantities of gas in the rectum and even the whole intestine before and during radiotherapy. These accumulations happen because the origins of gas are poorly understood.

In the first case (prostate cancer patient), we found that the rectal lumen still expanded significantly after the patient emptied his stool. There was no space between the intestinal wall and the target volume. After the prescribed dose was given, the volume dose of rectal $\mathrm{D} 2 \mathrm{~cm}^{3}$ was as high as $662 \mathrm{cGY}$. The patient had undergone pelvic radiotherapy in the past, so it was urgent to reduce the doses of rectal irradiation. During the three SBRT treatments, gas extraction ranged from 10 to $20 \mathrm{~mL}$ each time. The rectum $\mathrm{D} 2 \mathrm{~cm}^{3}$ volume was significantly reduced by $43 \%$, and the distance between the rectum and the target area was widened sharply. The maximum distance between the rectum and the target area was widened by about $2.14 \mathrm{~cm}$. This method dramatically reduces radiation exposure and doses to the rectum while being very safe and straightforward.

In SBRT of tumors near the rectum, sparing of the rectal wall is a prerequisite for the safe delivery of doses beyond $78 \mathrm{~Gy}$, as it is an organ at risk (9). However, rectal doses exceeding 70 Gy are predictors of rectal toxicity (10); therefore, it is very important to reduce the rectal radiation doses required. There are many clinical methods for reducing rectal doses, and each has its advantages and disadvantages. Spatial sparing of the rectum can be achieved by introducing a spacer material into the rectoprostatic space, thus separating the anterior rectal wall from the PTV (13-15). However, hyaluronate gel injection requires local anesthesia and intravenous sedoanalgesia, which is time-consuming and traumatic for the patients. Also, since tissues may become fibrotic after RT, it seems unreasonable that HGI would be meaningful and feasible in the reirradiation setting. HGI works by widening the distance between the rectum and the vagina or prostate to lower the rectal doses, but it does not address the problem of rectal volume caused by rectal inflation. If the patient has no previous history of pelvic radiotherapy, rectal aspiration plus hyaluronate gel injection may be the best option.

Among the two patients with gynecological diseases treated with HDR-BR, the second case had rectal flatulence. The transverse diameter of the intestinal cavity was larger than $5 \mathrm{~cm}$, which could not be alleviated by an oral laxative. After performing rectum aspiration on the patient, the patient was repositioned with CT, and the radiotherapy plan was customized to ensure that the patient received the highest prescribed dose with the lowest possible organ toxicity. The patient's rectal dose dropped by up to $20 \%$ using this method. Based on a conclusion drawn from these three patients, we believe that there is a positive correlation between the decrease in rectal dose and the amount of aspiration. However, the specific scope and degree still need to be verified with a larger number of patients. This part of the work is being collected and conducted clinically by our unit.

An intravaginal balloon catheter (16) was developed in 2004, which has decreased high-dose-rate-induced organ toxicity in the bladder and rectum. Nevertheless, in a randomized study of patients with cervical carcinoma treated with HDR-BT (17), one patient was reported to have a minor vaginal tear as a result of overinflation of the 
bladder-rectum spacer balloon. Furthermore, there are some other clinical methods, such as the use of volumetric modulated arc therapy (VMAT), to reduce rectal dose in radiotherapy for prostate cancer (18). However, the use of this method in reducing the dose is limited.

\section{Conclusions}

Our rectal gas extraction technique is feasible in reducing rectal toxicities during pelvic SBRT and HDR-BT.

\section{Acknowledgments}

Funding: This study was supported by a grant from the Natural Science Foundation of Zhejiang Province (grant number: LQ19H160033).

\section{Footnote}

Reporting Checklist: The authors have completed the Case Series reporting checklist. Available at http:// dx.doi.org/10.21037/apm-20-1063

Conflicts of Interest: All authors have completed the ICMJE uniform disclosure form (available at http:// dx.doi.org/10.21037/apm-20-1063). The authors have no conflicts of interest to declare.

Ethical Statement: The authors are accountable for all aspects of the work in ensuring that questions related to the accuracy or integrity of any part of the work are appropriately investigated and resolved. All procedures performed in studies involving human participants were in accordance with the ethical standards of the institutional and/or national research committee(s) and with the Helsinki Declaration (as revised in 2013). Written informed consent was obtained from the patient.

Open Access Statement: This is an Open Access article distributed in accordance with the Creative Commons Attribution-NonCommercial-NoDerivs 4.0 International License (CC BY-NC-ND 4.0), which permits the noncommercial replication and distribution of the article with the strict proviso that no changes or edits are made and the original work is properly cited (including links to both the formal publication through the relevant DOI and the license). See: https://creativecommons.org/licenses/by-nc-nd/4.0/.

\section{References}

1. Klement RJ, Guckenberger M, Alheid H, et al. Stereotactic body radiotherapy for oligo-metastatic liver disease Influence of pre-treatment chemotherapy and histology on local tumor control. Radiother Oncol 2017;123:227-33.

2. Onderdonk BE, Gutiontov SI, Chmura SJ. The Evolution (and Future) of Stereotactic Body Radiotherapy in the Treatment of Oligometastatic Disease. Hematol Oncol Clin North Am 2020;34:307-20.

3. Wang X, Liu R, Ma B, et al. High dose rate versus low dose rate intracavity brachytherapy for locally advanced uterine cervix cancer. Cochrane Database Syst Rev 2010;(7):CD007563.

4. Umezawa R, Murakami N, Nakamura S, et al. Imageguided interstitial high-dose-rate brachytherapy for locally recurrent uterine cervical cancer: A single-institution study. Brachytherapy 2018;17:368-76.

5. Ríos I, Rovirosa A, Ascaso C, et al. Vaginal-cuff control and toxicity results of a daily HDR brachytherapy schedule in endometrial cancer patients. Clin Transl Oncol 2016;18:925-30.

6. Banerjee R, Kamrava M. Brachytherapy in the treatment of cervical cancer: a review. Int J Womens Health 2014;6:555-64.

7. Logsdon MD, Eifel PJ. Figo IIIB squamous cell carcinoma of the cervix: an analysis of prognostic factors emphasizing the balance between external beam and intracavitary radiation therapy. Int J Radiat Oncol Biol Phys 1999;43:763-75.

8. Nakamura N, Hatanaka S, Takahashi O, et al. Gas in the rectum tends to reduce during radical external beam radiotherapy for localised prostate cancer. J Med Imaging Radiat Oncol 2014;58:253-6.

9. Spratt DE, Pei X, Yamada J, et al. Long-term survival and toxicity in patients treated with high-dose intensity modulated radiation therapy for localized prostate cancer. Int J Radiat Oncol Biol Phys 2013;85:686-92.

10. Marks LB, Yorke ED, Jackson A, et al. Use of normal tissue complication probability models in the clinic. Int $\mathrm{J}$ Radiat Oncol Biol Phys 2010;76:S10-9.

11. Su Z, Zhao T, Li Z, et al. Reduction of prostate intrafraction motion using gas-release rectal balloons. Med Phys 2012;39:5869-73.

12. Riley DS, Barber MS, Kienle GS, et al. CARE guidelines for case reports: explanation and elaboration document. J Clin Epidemiol 2017;89:218-35.

13. Kishi K, Mabuchi Y, Sonomura T, et al. Eradicative 
brachytherapy with hyaluronate gel injection into pararectal space in treatment of bulky vaginal stump recurrence of uterine cancer. J Radiat Res 2012;53:601-7.

14. Kashihara T, Murakami N, Tselis N, et al. Hyaluronate gel injection for rectum dose reduction in gynecologic highdose-rate brachytherapy: initial Japanese experience. J Radiat Res 2019;60:501-8.

15. Tang Q, Zhao F, Yu X, et al. The role of radioprotective spacers in clinical practice: a review. Quant Imaging Med Surg 2018;8:514-24.

16. Eng TY, Fuller CD, Cavanaugh SX, et al. Significant rectal and bladder dose reduction via utilization of Foley balloon catheters in high-dose-rate tandem and ovoid intracavitary brachytherapy of the uterine cervix. Int J Radiat Oncol Biol Phys 2004;59:174-8.

17. Rai B, Patel FD, Chakraborty S, et al. Bladder-Rectum Spacer Balloon versus Vaginal Gauze Packing in High Dose Rate Brachytherapy in Cervical Cancer: A Randomised Study. Part II. Clin Oncol (R Coll Radiol) 2015;27:713-9.

18. Soda R, Hatanaka S, Hariu M, et al. Evaluation of geometrical uncertainties on localized prostate radiotherapy of patients with bilateral metallic hip prostheses using 3D-CRT, IMRT and VMAT: A planning study. J Xray Sci Technol 2020;28:243-54.

Cite this article as: Jiang X, Chen Y, Liu J, Ning L, Sun X, Zheng L, Li X, Wang F, Zhu X, Lou Y, Yan S. Reduction in rectal doses of pelvic radiotherapy with excessive rectal gas extraction: a single-institution case report. Ann Palliat Med 2021;10(6):7062-7068. doi: 10.21037/apm-20-1063 